\title{
ACTIVIDADES ESTRATÉGICAS PARA FORTALECER EL SECTOR TURÍSTICO EN LA HEROICA CIUDAD DE HUAJUAPAN DE LEÓN, OAXACA
}

\author{
STRATEGIC ACTIVITIES TO STRENGTHEN THE TOURISM SECTOR IN \\ THE HEROIC CITY OF HUAJUAPAN DE LEÓN, OAXACA
}

\begin{abstract}
Lilia Alejandra Flores-Castillo
Universidad Tecnológica de la Mixteca, México

Missael Ruiz Corrales

Universidad Tecnológica de la Mixteca, México

Andrés Guzmán Méndez

Investigador independiente

Recibido: 18 de noviembre de 2019

Aceptado: 10 de enero de 2020

\section{RESUMEN}

Un destino turístico usualmente se puede definir como aquel lugar con riqueza natural de gran belleza; sin embargo, aunque localmente no se tenga una dotación de factores naturales favorable para el turismo como es el caso de la Heroica Ciudad de Huajuapan de León, Oaxaca, al existir diferentes modalidades de turismo, cabe la posibilidad de que, mediante la innovación, se desarrollen actividades atractivas con fines de esparcimiento en un marco turístico en el que se satisfagan las diversas expectativas del usuario. A partir de un estudio cualitativo, se plantea como objetivo principal presentar una serie de acciones estratégicas que permitan incentivar el desarrollo de actividades turísticas que generen un lugar atractivo, y por lo tanto, el fortalecimiento del sector turístico de la mencionada ciudad, cuya puesta en marcha depende del trabajo colectivo entre la población, empresas y gobierno.
\end{abstract}

Palabras clave: destino turístico, innovación, rutas turísticas, gobernanza y organización empresarial.

\begin{abstract}
Usually, a tourist destination can be defined as that place with natural and cultural resources of great beauty; however, although locally there is no endowment of natural factors favorable to tourism, such as in the Heroic City of Huajuapan de León, Oaxaca, as there are different types of tourism, it is possible that, through innovation, attractive activities for leisure purposes can be generated, in a tourist setting where the different expectations of
\end{abstract}

Este es un artículo Open Access bajo la licencia Creative Commons AtribuciónNoComercial-Compartirlgual 4.0 
the user are met. Based on a qualitative study, the main objective of this paper is to present a series of strategic actions that encourage the development of tourist activities that generate an attractive place, and therefore, the strengthening of the tourist sector of the aforementioned city, whose implementation depends on the collective work between the community, the business sector and the government.

Keywords: tourist destination, innovation, tourist routes, governance and business organization.

\section{Introducción}

El turismo se ha convertido en las últimas décadas en un fenómeno que tiene la capacidad de mover masas, con una gran importancia económica y una relevante participación en el desarrollo económico a través de la generación de fuentes de empleo y creación de nuevas empresas especializadas en prestar este servicio; se ha convertido en la principal fuente de ingresos de divisas para al menos 50 países en vías de desarrollo, entre ellos México, esto ha sucedido aún y cuando estos países presentan una participación pequeña en la industria mundial del turismo. Se argumenta que el turismo podría contribuir a solucionar algunos de los problemas de pobreza que enfrentan muchas regiones en países en vías de desarrollo (Altés, 2006).

Sumado a lo anterior, algunos estudios han comprobado que el desarrollo del sector turístico podría ser un factor que permita promover de manera indirecta el crecimiento económico de las regiones que desarrollan dicha actividad, primero al estimular el crecimiento de otros sectores, y segundo al incrementar el ingreso doméstico y la demanda efectiva; los estudios prueban la relación entre el desarrollo turístico y el crecimiento económico, y evidencian la importancia de la identificación de los factores potenciales que permitan el desarrollo del turismo (Gökavali y Bahar, 2006).

En primera instancia, es importante denotar que el concepto de turismo comprende un conjunto de modalidades; turismo de aventura, cultural, religioso, gastronómico, deportivo, histórico, ecoturismo, entre otras. Las cuales pueden adaptarse para la creación de un destino turístico con temáticas especificas o múltiples. Entendiendo con ello no solo el atractivo ligado a la existencia de enclaves y parajes naturales de gran belleza (Ejarque, 2016), sino aquellos lugares que han logrado generar mediante la innovación, actividades atractivas con fines de esparcimiento, en un marco turístico en el que satisfacen las diversas expectativas del usuario.

Bercial y Timón (2005) mencionan la importancia de desarrollar destinos turísticos complejos, sostenibles y competitivos. La competitividad se vincula con la idea de generar un servicio único, para lo cual la creatividad y la innovación juegan un papel fundamental en los determinantes de la oferta y en toda la cadena de valor (Porter, 1991). Con relación a lo antes mencionado, un consumidor más exigente e informado presiona al mercado turístico para que, mediante un proceso continuo de innovación, sean satisfechas sus necesidades actuales en cuanto a la demanda de servicios turísticos se refiere; por lo cual, es necesario percibir y entender los posibles cambios que generen una tendencia en la demanda turística, a través de determinar el perfil del consumidor, sus gustos, preferencias, edad, frecuencia de consumo, estatus social y cultural entre otras variantes. 
El turista, busca nuevas experiencias, propuestas temáticas y productos personalizables que le permitan relegar de manera momentánea sus actividades rutinarias. Sin duda, los conocimientos acerca de las tendencias en el mercado del turismo, dan la pauta no solo para la comprensión del comportamiento e intereses de los nuevos agentes usuarios del turismo, sino que además permiten buscar nuevas formas de sorprender a los turistas en una nueva modalidad (Ejarque, 2016).

La Heroica Ciudad de Huajuapan de León, Oaxaca, es una ciudad, que está en constante crecimiento; por lo tanto, es necesario crear un desarrollo local, que de acuerdo a la Comisión Económica para América Latina y el Caribe (Aghón, Alburquerque y Cortés, 2001), «es un proceso de transformación de la economía y la sociedad territorial orientado a superar las dificultades y exigencias del cambio estructural, la apertura y la globalización, con el fin de mejorar las condiciones de vida de la población local». Lo anterior sustenta la idea de replantear nuevas propuestas buscando innovar e impulsar el turismo en la Heroica Ciudad de Huajuapan de León.

Para el desarrollo de la propuesta planteada, en el presente trabajo de investigación, se formularon cuatro estrategias; la primera consiste en la creación de rutas turísticas, la segunda, en la creación de dos vallas publicitarias, la tercera, en actividades atractivas para los turistas y la cuarta, consiste en acciones estratégicas que deben realizar los actores implicados como el gobierno, los empresarios y la población.

\section{Desarrollo}

En la actualidad el turismo juega un papel muy importante a nivel mundial, México es uno de los países que se ha visto beneficiado por el desarrollo de la actividad turística, teniendo impacto en la generación de empleos y en el desarrollo local y regional, debido al gran número de viajeros que año tras año incrementa su visita al país provocando una fuerte derrama económica, lo que representa una exigencia de mayor demanda de destinos turísticos.

Entre los Estados con mayor demanda turística se puede citar en orden descendente a Quintana Roo, Ciudad de México, Jalisco, Guerrero y Veracruz (Secretaría de Turismo [SECTUR], 2016). El estado de Oaxaca ocupa el lugar decimotercero, concurrido por 2968064 turistas nacionales y 171382 turistas extranjeros durante el 2016. ${ }^{1}$

Oaxaca es un Estado que se ubica en el suroeste del país, colindando al norte con Puebla y Veracruz, al sur con el Océano Pacifico, al este con Chiapas, y al oeste con Guerrero (Enciclopedia de los Municipios y Delegaciones de México, 15 de agosto de 2018); es un Estado con gran oferta turística por su riqueza natural, cultural e histórica. Dentro de los atractivos turísticos que ofrece se encuentran las zonas arqueológicas, conventos del siglo XVI, templos barrocos, edificios civiles de la época colonial, museos, arte y cultura, fiestas religiosas con sus típicas calendas y mayordomías, tradiciones, playas y bahías, gastronomía, artesanías (alebrijes, textiles de lana, alfarería de barro, etc.) y su naturaleza (Soto y Brena, 2008, p. 3). El estado de Oaxaca cuenta con

1 Estos datos son los más recientes para cada uno de los Estados, de acuerdo a SECTUR (2016). 
sitios culturales declarados como patrimonio de la humanidad por la Organización de las Naciones Unidas para la Educación, la Ciencia y la Cultura (UNESCO), entre ellos, se encuentran el centro histórico de la ciudad, al igual que su zona arqueológica denominada Monte Albán, reconocidas el 11 de setiembre de 1987; mientras que en el 2010 se agregó a la lista las Cuevas Prehistóricas de Yagul y Mitla en los Valles Centrales (UNESCO, 9 de enero de 2020). De acuerdo con SECTUR del estado de Oaxaca (9 de enero de 2020), se tienen las siguientes rutas turísticas: 1. caminos de mezcal, 2. mágica de las artesanías, 3. de la fe Juquila, 4. Sierra Juárez, 5. de la costa Oaxaqueña, 6. de la Chinantla, 7. de la Mixteca, 8. del café Sierra Sur, 9. reserva de la biósfera, 10. del Istmo. Las rutas mencionadas permiten potencializar e impulsar todas las regiones que componen el estado de Oaxaca, de tal manera que no solo es beneficioso para el propio Estado, también lo es para los turistas, dado que pueden disfrutar de la riqueza gastronómica, artística y cultural que ofrece Oaxaca.

Entre los municipios que integran la Ruta de la Mixteca, se encuentra el municipio Heroica ciudad de Huajuapan de León, que está identificado como el centro comercial más importante de la región; y dada dicha importancia que es aunada también al hecho de ser la cabecera municipal ha presentado mayor índice de crecimiento económico, principalmente en actividades de comercio y servicios (Gobierno del estado de Oaxaca, 13 de octubre de 2017).

De acuerdo con Martínez (2001) y González (2011) Huajuapan de León es sin lugar a dudas una ciudad de relevancia histórica, de gran importancia por su participación en la Guerra de Independencia. Su relación histórica tiene lugar a partir del sitio llevado a cabo en dicha ciudad que tuvo una duración de 111 días.

Entre los héroes que resaltan en la historia de la ciudad se encuentran el general Antonio de León y Loyola, el coronel Valerio Trujano, el Indio de Nuyóo -valiente indígena que en el mencionado sitio hizo proezas de astucia y de valor- y Micaela Galindo. Otros personajes que se destacan en la ciudad, por su atribución son José López Alavez, músico y compositor de la «Canción Mixteca», quien ganó el primer lugar el 31 de marzo de 1918, también logró un segundo lugar con la canción «Apasionada». Asimismo, Antonio Martínez Corro, es considerado un distinguido habitante de la región, reconocido por las aportaciones que sus obras han traído al desarrollo y preservación de la cultura Mixteca; dentro de las más reconocidas e importantes se encuentran su arreglo del famoso Jarabe Mixteco, un Himno a Cristo Rey que los católicos cantan desde 1929, la Suite Oaxaqueña y otras de la cultura popular (Mendoza, 1992).

Adicionalmente al hecho histórico ya mencionado, se tienen importantes sitios arqueológicos que representan lugares prehispánicos en los que tuvo presencia la cultura mixteca.

Guzmán, Flores y Reyes (2019) realizaron un diagnóstico para identificar los atractivos de la ciudad y sus alrededores, la oferta, la demanda y el potencial turístico con el que cuenta; concluyendo que la Heroica Ciudad de Huajuapan de León, Oaxaca, no está considerada y posicionada como un destino turístico, pero cuenta con recursos culturales, patrimoniales, antropológicos, religiosos, gastronómicos e históricos que se pueden estimar y aprovechar para crear un producto competitivo y posicionarse exitosamente en el mercado turístico regional. En lo que respecta a las oportunidades de las modalidades turísticas que se pueden desarrollar en la región se mencionan el turismo cultural, histórico, de aventura, religioso, gastronómico, deportivo y ecoturismo. 
En este sentido, en la presente investigación se plantea la identificación de acciones que permitan incentivar el turismo; teniendo como objetivo primario definir cuatro estrategias que consideren acciones pertinentes de acuerdo a las características de la ciudad. La metodología seguida es de tipo cualitativo con diseño descriptivo. Se inició con una revisión documental sobre los antecedentes de la ciudad en las referencias cronológicas que se encuentran en la biblioteca central de la localidad; así como la consulta en la base de datos de la Dirección de Registro Público de Monumentos y Zonas Arqueológicas del Instituto Nacional de Antropología e Historia. De igual manera, se examinó el inventario de la Regiduría de Fomento Económico y Turismo y de la Dirección de Monumentos Históricos, complementando esta información mediante entrevistas a profundidad a los encargados de las instituciones mencionadas y recopilación de fotografías de los posibles lugares con atractivo turístico. Se determinó como objetivo identificar los posibles atractivos, recursos culturales, gastronómicos, religiosos, patrimoniales, antropológicos, actividades recreativas y servicios turísticos existentes.

Aunado a lo anterior, se diseñó y aplicó un instrumento de recolección de información primaria que se aplicó a los visitantes y turistas siguiendo un tipo de muestreo a juicio. El trabajo de campo se realizó en los hoteles principales así como en el zócalo de la ciudad, sujeto a la disponibilidad de los entrevistados para participar en la encuesta, durante distintos periodos de tiempo vacacional (vacacionales de Semana Santa²) y durante las fechas de celebraciones de algunas festividades patronales y locales como el festejo en honor al Señor de los Corazones.

\section{Resultados}

Del análisis de la información primaria y secundaria se concluye necesario impulsar el turismo en la ciudad, por lo tanto, como resultado se plantea una propuesta de acciones enfocadas al sector turístico conformada por cuatro estrategias. La primera consiste en la creación de rutas turísticas, la segunda en la creación de dos vallas publicitarias, la tercera en actividades atractivas para los turistas y la cuarta consiste en acciones estratégicas y específicas que deben realizar los actores implicados como son el gobierno, los empresarios y la población.

\section{Creación de rutas turísticas}

Se propone la creación de rutas turísticas ${ }^{3}$ sustentado en el hecho de que dentro de las modalidades turísticas que se pueden desarrollar dentro de las región, pueden considerarse el turismo cultural o religioso. Los monumentos conmemorativos, históricos, artísticos y zonas arqueológicas considerados por la Dirección de Monumentos Históricos y la Regiduría de Fomento Económico y Turismo de la Heroica Ciudad de Huajuapan de León, además fueron considerados los lugares que los turistas expresaron les gustaría visitar por ser de su interés en esta propuesta de rutas turísticas.

2 Se realizó la encuesta durante este periodo vacacional ya que de acuerdo con la Regiduría de Fomento Económico y Turismo hay gran variedad de actividades, tanto en el municipio de Huajuapan como en los pueblos que se encuentra a su alrededor; el primer viernes, se celebra en San Francisco Yosocuta y en Agua Dulce; el segundo viernes, se celebra en San Miguel Papalutla y en Huajuapan de León; el tercer viernes, se celebra en Asunción Cuyotepeji y Santa María Yuxichi; el cuarto viernes, Tezoatlan de Segura y Luna y en Santa María Ayú; el quinto viernes, se celebra en la colonia El Calvario, de Huajuapan de León y en Ahuehuetitlan de González; el sexto viernes, se celebra en Santiago Chilixtlahuaca; y finalmente en la Semana Santa se celebra en Santiago Huajolotitlan, San Sebastián Progreso, también parroquias y agencias de Huajuapan de León, Oaxaca.

3 Una ruta turística consiste en «Un circuito temático o geográfico que se basa en un patrimonio natural o cultural de una zona y se marca sobre el terreno 0 aparece en los mapas» (Diario Oficial de la Federación [DOF], 2016). Cabe mencionar que Huajuapan de León pertenece a la ruta Museológica. 
En la Tabla 1 se muestra la propuesta de cada una de las seis rutas turísticas pensadas para cumplir con el objetivo de fomento al turismo y promoción y difusión de los sitios más emblemáticos y representativos de la ciudad. Las rutas se conforman teniendo como inicio y termino del recorrido el centro de la ciudad, con una duración de un día.

\section{Tabla 1}

Rutas turísticas en un tranvía

\section{Ruta 1}

- Monumento al General Antonio de León

- Sagrario Diocesano

- Catedral a San Juan Bautista

- Museo Regional de Huajuapan (MUREH)

- Monumento al Indio de Nuyoo

- Cerro de las Minas

- Casa de la Pólvora

- Balneario los girasoles

- Kiosco del Parque Independencia

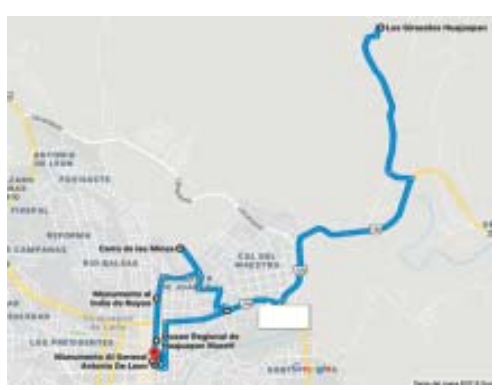

\section{Ruta 2}

- Monumento a José López Alavés

- Casa de la cultura «Antonio Martinez Corro»

- Monumento al General Lázaro Cárdenas del Río

- Lienzo Charro de Huajuapan de León

- Paraje Salto del Agua o Salto del Tigre

- El Boquerón de Santo Domingo Tonalá

- Parque Jagüey

- Kiosco del Parque Independencia

\section{Ruta 3}

- Coronel Valerio Trujano

- Palacio Municipal

- Jardín Etnobotánico

- Monumento a la Constitución Federal

- Monumento a los Niños Héroes

- Paseo de las campanas

- UTM

- Visita a una clínica o spa

- Kiosco del Parque Independencia

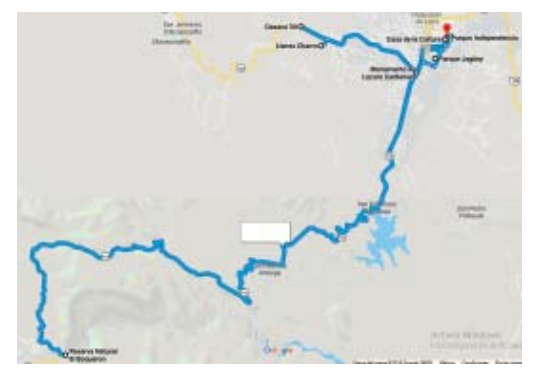

\section{Ruta 4}

- Monumento al General Antonio de León

- Sagrario Diocesano

- Catedral a San Juan Bautista

- Museo Regional de Huajuapan (MUREH)

- Hemiciclo a Juárez

- Monumento al «Jarabe Mixteco»

- Zona arqueológica de Santa Teresa

- Balneario

- Visita a una clínica o spa

- Kiosco del Parque Independencia
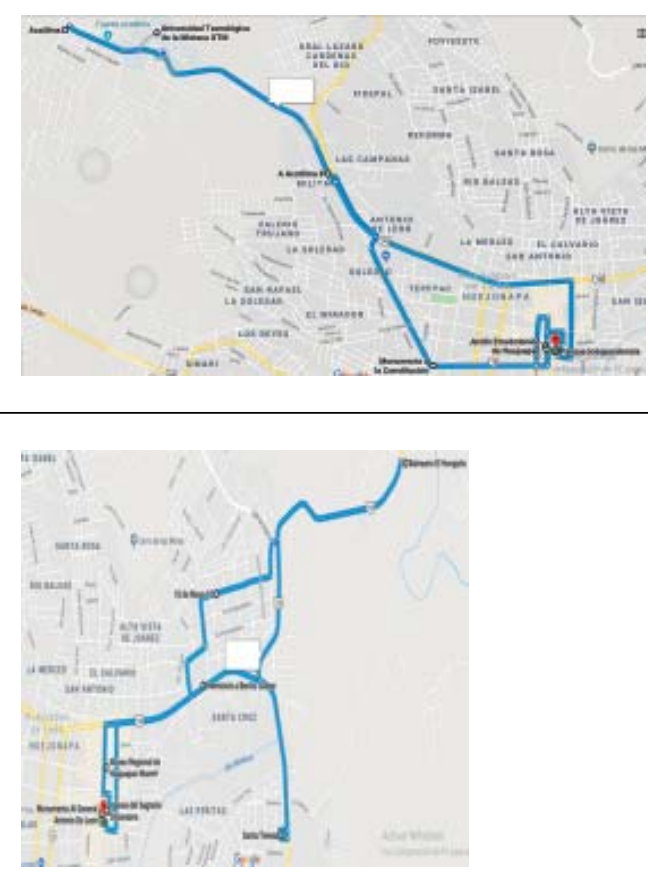


\section{Ruta 5}

- Monumento a José López Alavés

- Casa de la cultura «Antonio Martínez Corro»

- Monumento al General Lázaro Cárdenas del Río

- Presa «Lázaro Cárdenas» o San Francisco Yosocuta

- Visita a una clínica o spa

- Deportivo el Carmen

- Kiosco del Parque Independencia

\section{Ruta 6}

- Monumento al General Antonio de León

- Sagrario Diocesano

- Catedral a San Juan Bautista

- Museo Regional de Huajuapan (MUREH)

- Hemiciclo a Juárez

- Monumento al «Jarabe Mixteco»

- Andador de Santa María Yuxichi

- Balneario

- Kiosco del Parque Independencia

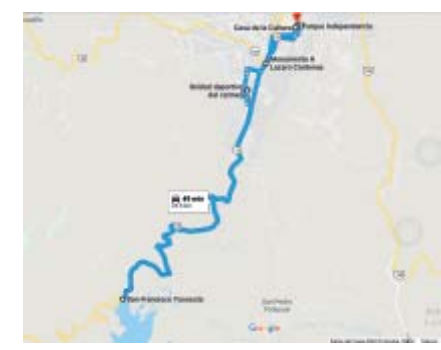

Nota. Imágenes de INEGI.

Las rutas mencionadas deben cumplir algunas especificaciones, por ejemplo, una transportadora turística ${ }^{4}$ terrestre, como un tranvía para recorridos turísticos (ver Figura 1) que incluya en su lado derecho la imagen del cerro de las minas y del lado izquierdo el de la pareja del jarabe mixteco; además, en la parte posterior debe mostrar el logo de la feria de la ciudad de Huajuapan de León, este se renovaría cada año.

\section{Figura 1}

Tranvía para las rutas turísticas

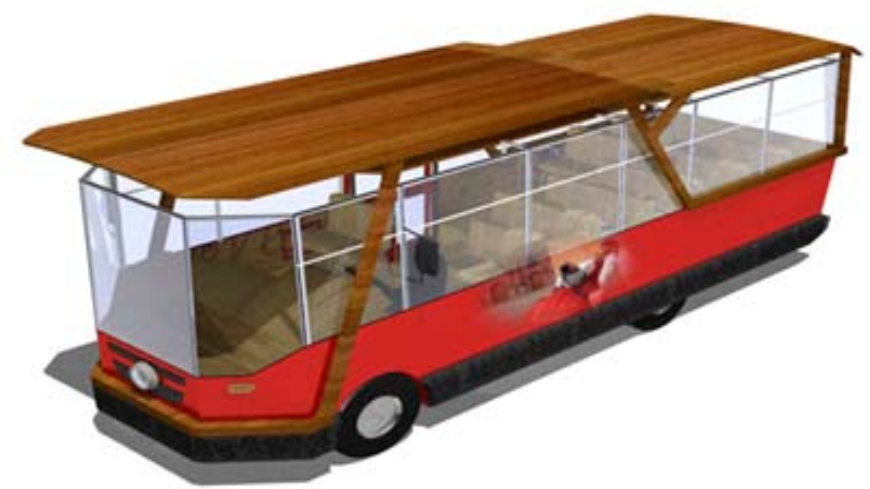

Nota Obtenida en Noriega (2008).

4 Es «aquel que proporciona el servicio de traslado de personas con fines recreativos, culturales, de esparcimiento o de negocios a un destino específico» (DOF, 2016). 
La transportadora turística, además de las especificaciones ya mencionadas, debe incluir sistemas de comunicación que permitan al guía de turistas ${ }^{5}$ comunicarse con los visitantes y extender datos de relevancia de cada uno de los sitios visitados. Dicho guía debe obtener una credencial expedida por SECTUR siguiendo el Reglamento de la Ley General de Turismo publicado en el DOF (Cámara de Diputados del H. Congreso de la Unión, 6 de julio de 2015) que menciona en el artículo 79, los siguientes requisitos:

I. Dirigir la solicitud a la Secretaría, a través de los formatos que ésta determine;

II. Acreditar su nacionalidad. En caso de ser extranjeros, además, deberán demostrar fehacientemente su legal estancia en el país, así como la condición migratoria que les permita desarrollar la actividad de Guía de Turistas;

III. Acreditar sus estudios o el proceso de formación que avalen los conocimientos, habilidades y destrezas necesarias para desarrollar profesionalmente la actividad de Guía de Turistas;

IV. Demostrar el dominio del idioma español en las habilidades de lectura, habla, escritura y entendimiento del idioma;

V. Presentar identificación oficial;

VI. Presentar el Registro Federal de Contribuyentes y Clave Única del Registro de Población;

VII. Presentar un comprobante de domicilio, y

VIII. Demostrar que cumple con los demás requisitos establecidos en las Normas Oficiales Mexicanas aplicables a la materia.

La Secretaría determina los conocimientos y condiciones que debe reunir el solicitante para que, en su caso, se determine la especialización de sus conocimientos como guía de turista.

El titular de la Secretaría determina, mediante acuerdo que debe publicarse en el Diario Oficial de la Federación, los formatos a que se refiere la fracción I de este artículo, así como los conocimientos y condiciones que se requieren para alguna especialización como guías de turistas, además de los requisitos técnicos que permiten al solicitante acreditar y demostrar la información requerida.

Sumado a los requisitos, antes mencionados, debe tener conocimientos generales de la ciudad de Huajuapan de León, de primeros auxilios; dominio del idioma extranjero (inglés) y nativo (lengua mixteca).

Son todas aquellas «personas físicas que proporcionan al Turista nacional o extranjero orientación e información profesional sobre el patrimonio cultural, natural y, en general, la relativa a los Atractivos, Destinos, Regiones y Servicios Turísticos» (Cámara de Diputados del H. Congreso de la Unión, 6 de julio de 2015); cabe señalar que la Regiduría de Fomento Económico y Turismo cuenta con una persona indicada que realiza recorridos. 


\section{Creación de vallas publicitarias}

En esta estrategia se propone la realización de por lo menos dos vallas publicitarias ${ }^{6}$ en el Parque «Independencia» con las siguientes características: la valla debe medir 2.20 metros de largo por 1.60 de ancho, sin considerar los tubos enterrados que sostienen la valla. Esta valla tiene dos vistas: la primera vista, en la parte superior un mapa con las rutas con las que cuenta la ciudad; y dentro del mapa marcado con símbolos o números lugares de interés o destinos atractivos, por ejemplo, balnearios, monumentos, zonas arqueológicas, deportivos, mercados, hospitales y algún lugar de entretenimiento; en la parte inferior los nombres de cada uno de ellos con sus símbolo o número.

La segunda vista, debe contener un directorio de servicios de emergencia como la Comisión Nacional de Emergencia, Cruz Roja, Bomberos, Policía Municipal, la Procuraduría General de la República (PGR), Palacio Municipal, información de algún hospital o clínica (pública), etc.

\section{Actividades atractivas para los turistas}

Esta estrategia tiene la finalidad de incluir actividades recreativas con el propósito de dar respuesta a las preferencias que los turistas expresaron en el instrumento de recolección de información; por lo tanto, estas actividades deben impulsarse dentro de la ciudad. Los porcentajes para cada actividad corresponden a las respuestas de los entrevistados: recorridos por la naturaleza (73.47\%), tirolesa (51.02\%), ferias populares (48.9\%), escalada, montañismo (45.92\%), festivales de música (45.92\%), recorrido con todo terreno (42.86\%), exposición gastronómica (41.84\%), visita a galerías de artesanías (40.82\%), bicicleta (recorridos) (40.82\%), parques temáticos para niños (37.76\%), globos aerostáticos (37.76\%), museos de ciencia y tecnología (37.76\%), acontecimientos deportivos, atletismo, carrera de coches y motos (37.76\%), visitas a industria de mezcal (37.76\%), jardines botánicos (36.73\%) y visita a galerías de arte (35.71\%). En la Tabla 2 se especifican las siguientes actividades a desarrollar en la ciudad.

6 Cabe mencionar que las vallas publicitarias forman parte de un instrumento de publicidad y no como una campaña publicitaria. 
Tabla 2

Actividades propuestas para fomentar el turismo de acuerdo a las tendencias

\begin{tabular}{|c|c|}
\hline Tendencias & Actividades \\
\hline Experiencia de usuario & $\begin{array}{l}\text { - } \quad \text { Creación de talleres, con el propósito de que los turistas puedan crear su propio recuerdo } \\
\text { de la ciudad. } \\
\text { - } \quad \text { Llevar a los turistas a la hora que se oculta el sol en el cerro de las Minas para soltar } \\
\text { globos de cantolla y se les narren historias o leyendas de la ciudad. } \\
\text { - } \quad \text { Vestir y caracterizar a los turistas con trajes tradicionales de la ciudad (como de la pareja } \\
\text { mixteca y de los matachines) y llevarlos al centro de la ciudad para participar en eventos } \\
\text { de musica y de baile. } \\
\text { - } \quad \text { Realizar un evento cultural donde se expongan las principales artesanías de la región. } \\
\text { - } \quad \text { Crear un festival de burbujas y de papalote (para los niños). } \\
\text { - } \quad \text { Impulsar el servicio de globos aerostáticos. } \\
\text { - } \quad \text { Creación de tirolesa o teleférico entre el cerro Yucunitza y el cerro de las Minas. } \\
\text { - } \quad \text { Feria del trueque (donde se puedan intercambiar cosas, aparatos electrónicos, ropa, } \\
\text { accesorios, mercancías, etc.). } \\
\text { - Creación de un concierto de Jazz. } \\
\text { - } \quad \text { Aumentar la realización de eventos musicales. } \\
\text { - } \quad \text { Incrementar las galerías de pinturas y obras que se realizan en la región. }\end{array}$ \\
\hline Sustentabilidad & $\begin{array}{l}\text { - } \quad \text { Creación de fiestas nocturnas o campamentos en balnearios de la ciudad. } \\
\text { - } \quad \text { Caminatas o recorridos en caballo por los principales cerros de la ciudad. } \\
\text { - } \quad \text { Crear carreras alrededor o mediante las principales vialidades de la ciudad. } \\
\text { - }\end{array}$ \\
\hline Destinos & Representación del hecho histórico de Huajuapan de León. \\
\hline Tecnología & $\begin{array}{l}\text { - Aplicación que cuenta la historia de Huajuapan de León. } \\
\text { - Creación de una aplicación donde se especifican las rutas turísticas y donde se } \\
\text { proporcionen información relevante de la ciudad (festival, calenda, noticia, etc.). } \\
\text { - Crear la feria de aparatos tecnológicos. }\end{array}$ \\
\hline Transporte & $\begin{array}{l}\text { - } \quad \text { Crear un vagón de trencito turístico. } \\
\text { - } \quad \text { Impulsar nuevamente vuelos en avionetas. }\end{array}$ \\
\hline
\end{tabular}

Nota. Basado en las encuestas realizadas a los turistas.

Cabe señalar que estas actividades ya se realizaban, por lo tanto, se busca retomar, pero a precios accesible para los turistas.

Reanudar el Bici-club, anteriormente consistía en recorridos en bicicleta apoyados de policías de seguridad. 


\section{Gobernanza y organización empresarial}

La estrategia consiste en la organización que contemple especialmente la colaboración entre población, empresas y Gobierno, con el objetivo de crear un consenso entre el número de participantes y actores (públicoprivado y sociedad civil) y así ejecutar estas estrategias de forma secuencial. Lo que permite definir el papel o las acciones que deben realizar cada uno de los actores implicados.

En la actualidad el sector turístico no representa una parte significativa de la economía local de la ciudad, debido a ello, este sería un factor para que las empresas no muestren interés por realizar erogaciones económicas en las primeras tres propuestas. Por consiguiente, el actor principal de inversión, en el corto plazo, puede ser el Gobierno a través de la Regiduría de Fomento Económico y Turismo, y también, se haría cargo de la coordinación y la organización de los responsables de los servicios públicos, de manera que se puedan mejorar algunos aspectos importantes para el desarrollo turístico.

Para que se efectúe la primera estrategia es necesario contar con la participación del Gobierno realizando mejoras en la infraestructura y en el mantenimiento de los lugares de interés. ${ }^{9}$

En el caso de la segunda estrategia, vallas publicitarias, su funcionamiento es posible con el apoyo de los patrocinadores que encuentre la regiduría; pueden ser centros comerciales o tiendas, grupos financieros como bancos y cajas de ahorro, etc., los cuales pueden hacer contribuciones en especie o en efectivo.

Para la tercera estrategia es necesario que el gobierno realice mejoras en la infraestructura de negocios y centros culturales (apertura de un centro de convenciones con sala de exhibiciones y diferentes auditorios, la conversión de espacios en desuso en museos y la apertura de centros comerciales, entre otros); además promover que las empresas asuman la conducción de alguna de estas actividades.

Finalmente, con el apoyo de las empresas de transporte se puede realizar un medio de control para monitorear el flujo de excursionistas, visitantes y turistas, mediante boletos de colores, en el que pueden participar los empresarios con patrocinio para la visita de sus negocios (alguna bebida, descuento del 10\%, etc.). Cabe señalar que por medio de la capacitación y asesoría de la Cámara de Comercio de Huajuapan de León y del Instituto de Capacitación y Productividad para el Trabajo del Estado de Oaxaca del municipio, los empresarios tienen la posibilidad de mejorar el servicio de atención a los turistas o visitantes, así como construir proyectos innovadores relacionados con este sector.

\section{Conclusiones}

Impulsar el sector turístico es fundamental para el desarrollo y competitividad regional, es mediante la innovación que se puede fortalecer y crear actividad turística en donde no la hay. En este sentido, las alianzas estratégicas entre los sectores empresariales, gubernamentales y sociedad civil resultan ser factores clave en las oportunidades que tiene la ciudad con relación al sector turístico. Por medio de inversiones en infraestructura,

9 Por ejemplo, en el caso de los monumentos que se encuentran grafitiados, las zonas arqueológicas descuidadas y la presa Yosocuta contaminada, etc. 
de programas gubernamentales que aporten a la ciudad en una mejora del transito vial, seguridad, cuidado y difusión de los lugares que tiene la ciudad de Huajuapan de León y sus alrededores, así como la debida exploración de las zonas arqueológicas, se puede incentivar las actividades turísticas. Logrando así cambiar la imagen que se tiene de una ciudad comercial por la de un destino turístico atrayente para los visitantes, quienes la tomarán como una de sus opciones al momento de viajar.

\section{Referencias}

Aghón, G., Alburquerque, F. y Cortés, P. (2001). Desarrollo económico local y descentralización en América Latina: Análisis comparativo. Comisión Económica para América Latina y el Caribe (CEPAL).

Altés, C. (2006). El turismo en América Latina y el Caribe y la experiencia del BID. Banco Interamericano de Desarrollo, Departamento de Desarrollo Sostenible.

Bercial, R. y Timón, D. A. (2005). Nuevas Tendencias en el desarrollo de destinos turísticos: marcos conceptuales y operativos para su planificación y gestión. Cuadernos de turismo, (15), 27-43.

Cámara de Diputados del H. Congreso de la Unión. (2015, 6 de julio). Reglamento de la Ley General del Turismo (art. 2, frac. XVII). Publicado en el Diario Oficial de la Federación. http://www.sectur.gob.mx/pdf/0216/Reglamento_Ley_General_Turismo.pdf

Diario Oficial de la Federación (DOF). (2016). Secretaría de Turismo. http://www.sectur.gob.mx/pdf/ACUERDO\%20CATA\%CC\% 81LOGO.pdf

Ejarque, J. (2016). Marketing y gestión de destinos turísticos nuevos modelos y estrategias 2.0. Madrid: Pirámide.

Enciclopedia de los Municipios y Delegaciones de México. (2018, 15 de agosto). Oaxaca. http://www.inafed.gob.mx/work/enciclopedia/ EMM20oaxaca/mediofisico.html

Gobierno del estado de Oaxaca. (2017, 13 de octubre). Plan Municipal de Desarrollo de Huajuapan de León 2014-2016. Turismo. http:// sisplade.oaxaca.gob.mx/indicadorescoplade/planes_municipales/2014_2016/039.pdf

Gökavali, U. y Bahar, O. (2006). Contribution of Tourism to Economic Growth: A Panel Data Approach. Anatolia, 17(2), 155-167. https:/ /doi.org/10.1080/13032917.2006.9687184

Gonzalez, L. (2011). El Huajuapan de ayer. Raíces.

Google. (s. f.). Mapa de Huajuapan de León, Oaxaca en Google maps. https://www.google.com.mx/maps/place/ Huajuapan+de+Le\%C3\%B3n,+Oax./@17.8047734,-97.8177176,13z/data=!3m1!4b1!4m5!3m4!1s0x85c6020679a780a3:0xb826a 39b58aceb9a!8m2!3d17.8112504!4d-97.7803482

Guzmán, A., Flores, L. A. y Reyes, P. (2019). Patrimonio cultural potencial turístico en la Heroica Ciudad de Huajuapan de León, Oaxaca. Revista de Investigación Académica Sin Frontera: División de Ciencias Económicas y Sociales, (29). http:// revistainvestigacionacademicasinfrontera.com/sistema/index.php/RDIASF/article/view/215

Martínez, L. G. (2001). Huajuapan ayer y hoy hechos y cosas de la Heroica Ciudad. Oaxaca de Juárez: Aurea de la nueva era.

Mendoza, T. (1992). Monografía del distrito de Huajuapan. Oaxaca: Colección Glifo.

Noriega, D. (2008). Maxei. https://danielanoriega.wordpress.com/2008/11/18/maxei/

Organización de las Naciones Unidas para la Educación, la Ciencia y la Cultura (UNESCO). (2020, 9 de enero). Lista del patrimonio mundial. http://whc.unesco.org/en/list/\&order=country\#alphaM

Porter, M. E. (1991). La ventaja competitiva de las naciones (vol. 1025). Buenos Aires: Vergara.

Secretaría de Turismo (SECTUR). (2016). Información turística por entidad federativa. http://www.datatur.sectur.gob.mx/SitePages/ InfTurxEdo.aspx

Soto, A. L. y Brena, R. G. (2008). Turismo y Economía en el Estado de Oaxaca. Turismo y Desarrollo Local, 1 (3), 3. 


\section{Lilia Alejandra Flores-Castillo}

Doctorado en Ciencias Económicas, profesor investigador de tiempo completo en la Universidad Tecnológica de la Mixteca. Publicaciones más recientes: Panorama de la MiPyME en el estado de Oaxaca; Patrimonio cultural potencial turístico en la Heroica Ciudad de Huajuapan de León, Oaxaca; La nueva oferta sobre las inversiones inmobiliarias (Fibras y Sibras) y su coyuntura hacia los mercados de especulación global, y su relación intrínseca con los créditos sindicados de las viviendas de interés social.

floresaly22@hotmail.com

ORCID: http://orcid.org/0000-0002-1345-5093

\section{Missael Ruiz Corrales}

Doctorado en Ciencias Administrativas. Profesor Investigador de tiempo completo de la Universidad Tecnológica de la Mixteca. Publicaciones recientes: Cuarta revolución industrial: Tecnologías en las áreas administrativas, contables, informáticas y de negocios; Modelo de gestión del conocimiento apoyado en las tecnologías de información y comunicación; Indicadores de confianza ciudadana como medida del desempeño y efectividad de las instituciones gubernamentales, un caso mexicano.

missael@mixteco.utm.mx

ORCID: http://orcid.org/0000-0003-2190-4597

Andrés Guzmán Méndez

Licenciado en Ciencias Empresariales por la Universidad Tecnológica de la Mixteca.

gmandres0@gmail.com

ORCID: http://orcid.org/0000-0002-2110-2319 